\title{
The study of dynamic characteristics during the vibration processing of parts
}

\author{
Elena Levchenko ${ }^{1, *}$ \\ ${ }^{1}$ Sevastopol State University, Department of Mechanical Engineering, 299053 Sevastopol, Russia
}

\begin{abstract}
The results of theoretical studies on the dynamic characteristics of the process of vibroprocessing are presented, the essence of which is to create conservative zones limited by the current lines of a certain fixed vector field, which contributes to the formation of a macrodomain structure in the working medium. Domain structuring of the working tool reflects the configuration of the field grid of the external dynamic field and is the basis of the technology of vibration processing.
\end{abstract}

\section{Introduction}

Vibration processing is widely used for various parts, and primarily for those with complex shapes, but there are limitations associated primarily with the size of the parts, that is fine flat and long parts. The first ones - due to the fact that during processing with the use of water or solutions of reactive substances, the parts adhere to the walls of the container and are collected in bags (this phenomenon disrupts the processing process, reduces the quality of processing of small-sized planar parts), the second ones - because of their uneven processing, associated with the complexity of ensuring a stable circulation movement in long vibrating containers. Therefore, it is necessary to search for new technological solutions for the application of vibration processing of such parts [1-5].

In this regard, the important scientific and practical tasks of developing a model of the process of the movement of parts and individual granules in a vibrational field, of introduction of methods for improving the process of vibratory processing by creating a design and technological system, taking into account the location of the vibration generator with respect to the container, choice of the container shape, the container lining, the abrasive tool, and also of expansion of possibilities of this method due to effective processing of fine flat and long parts are solved. The essence of vibration treatment consists in the mechanical or chemical-mechanical process of removing the smallest metal particles from the treated surface, as well as smoothing the microdeformation by their plastic deformation.

Processing directly represents the process of successive exposure to the surface of the workpiece of abrasive grains interspersed with the surface of special working granules (a binder of some form with a certain amount of abrasive grains embedded into it). This exposure (as a result of the interaction of the working granule with the workpiece) is a consequence of the action of force pulses from the walls of the oscillating container. In this case, dynamic contact of abrasive grains of the working granule with the surface of the

\footnotetext{
* Corresponding author: ealev1978@mail.ru
} 
workpiece takes place, leading to microcutting (during slipping) and dyeing of the surface material (with successive impacts). This interaction of the working granules with the surface of the workpiece has been thoroughly studied qualitatively, which allows us to state the existence at the present time of the generally accepted understanding of the nature of the mechanical processes of processing the surface of the workpiece with the working granule under vibration processing [6-8].

\section{Main text}

When processing under the same conditions, the power expended on the process can unambiguously characterize its productivity, since it is a common parameter for the whole variety of its technological operations, characterized by different final technological results, which in turn depend on a combination of a variety of factors also different for each operation [3].

Let the relationship between the power spent on the processing process and the productivity of this process be defined.

On account of the dissipative Rayleigh function, the developed mathematical model allows to determine the average useful power for a period depending on the parameters of the vibratory machine. In this case, the equation can be written in the form:

$$
N=\frac{1}{2 T} \int_{0}^{T}\left[B_{r}\left(\dot{x}_{r}(\omega, r, t)-\dot{x}(\omega, r, t)\right)^{2}+B_{r}\left(\dot{y}_{r}(\omega, r, t)+\dot{y}(\omega, r, t)\right)^{2}\right] d t,
$$

where $\omega$ and $r$ - are the angular velocity and eccentricity, respectively; $T-$ is the period defined as $\frac{2 \pi}{\omega}$.

This will determine the effectiveness of the application of a particular frequency and amplitude mode, but the dependence of the processing capacity on the location of the vibration generator cannot be determined in this way. This is related to the peculiarities of the chosen calculation scheme.

The power dissipated in the working medium will be proportional to the square of the difference in the velocities of the walls of the container and the layer of the working medium. Then the dependence of the useful power on the location of the vibration generator can be represented as:

$$
N=\frac{1}{l} \int_{S} \int_{0}^{T}\left[\frac{1}{2 T} B_{r}\left(\dot{x}_{r}(t)-\dot{x}_{p}(t)\right)^{2}+\frac{1}{2 T} B_{r}\left(\dot{y}_{r}(t)+\dot{y}_{p}(t)\right)^{2}\right] d t d s,
$$

where $S$ - is the function describing the geometric shape of the container through which the integration takes place and whose values are substituted during calculation of the velocity functions of the displacement of the container points $\dot{x}_{p}$ and $\dot{y}_{p}$, and which are determined by:

$$
\begin{aligned}
& x_{p}\left(x_{0}, y_{0}, t\right)=\dot{x}(t)+\sqrt{x_{0}^{2}+y_{0}^{2}} \cdot \dot{\phi}(t) \cos \left(\phi(t)+\operatorname{arctg}\left(\frac{x_{0}}{y_{0}}\right)\right) ; \\
& y_{p}\left(x_{0}, y_{0}, t\right)=\dot{y}(t)-\sqrt{x_{0}^{2}+y_{0}^{2}} \cdot \dot{\phi}(t) \sin \left(\phi(t)+\operatorname{arctg}\left(\frac{x_{0}}{y_{0}}\right)\right),
\end{aligned}
$$


where $l$ - is the length of the curve approximating the cross-section line of the container.

Coordinates of the location of the vibration generator, indirectly indicated in formula (1), are used in determining the velocities $\dot{x}_{r}, \dot{y}_{r}, \dot{x}_{p}$ and $\dot{y}_{p}$.

The results of calculations of the power transmitted to the working medium, depending on the location of the vibration generator, are presented in Fig. 1.

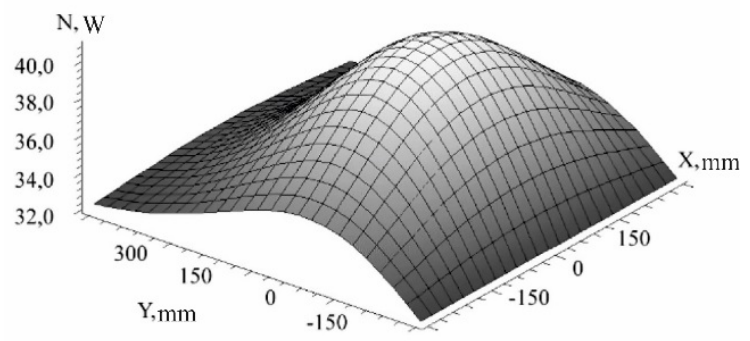

(a)

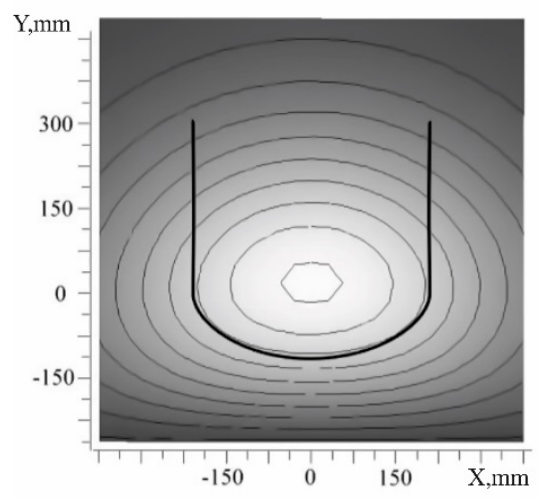

(b)

Fig. 1. Calculation of the average useful power transmitted by the walls of the container to the working medium for a period (depending on the location of the vibration generator) ( $a, b$ image in a three- and two-dimensional coordinate system, respectively)

It can be seen from the graphs that the (Fig. 1) location of the vibration generator in the center of mass of the container of the vibratory machine (coordinates - $0 ; 0$ ) is the most efficient. Accordingly, the useful power increases with the approach to this point. The advantages of using a circular trajectory, as well as a trajectory that is a vertically elongated ellipse, were given in work [11], which confirms the correctness of the chosen direction.

A further rise of the vibration generator results in a gradual decrease in power. Nevertheless, when installing the vibration generator under the bottom of the container, the processing capacity, on account of the calculated data, based on which the graphs are plotted, decreases significantly more abrupt [9-11].

It should be mentioned that the location of the vibration generator in the center is associated with some technical nuances and can be realized in four ways:

1. Location of two synchronized vibration generators;

2. Installation of two interconnected containers and placement of a vibration generator between them;

3. Placement of the vibration generator within the container (acceptable only for large containers, otherwise the working volume will be significantly reduced).

4. The weighting of the oscillatory system by securing the load under the vibration generator, that is, the displacement of the center of gravity, closer to the point of application of the disturbing force (this can be realized only on vibratory machines with small-volume containers, this also entails the need to increase the engine power).

It should be noted that conclusions about the efficiency of the location of the vibration generator with respect to the container must be made, taking into account also such a factor as the existence of a stable circulation movement, which is an important element contributing to the increase in processing capacity [10].

Below are the calculated values of the circulating velocity depending on the location of the vibration generator (Fig. 2) and the stability of the circulation motion (Fig. 3), determined by the root-mean-square deviation of the value of the working medium element displacement relating to the container wall. 
It follows from the data shown in the graph of the dependence of the circulation velocity on (Fig. 2) that with removal of the vibration generator into the lower right corner, the circulation velocity increases significantly.

If the vibration generator is located in the center of the container, the circulation velocity will be absent, that is, despite the fact that in a certain interval the working medium will move relative to the container walls, the average value of the displacement will be close to 0 . When the vibration generator is located in the upper left corner, the graph displays the negative circulation speed, that is, the working medium must rotate in the other direction (Fig 3). The displacement of the vibration generator upwards and to the left increases the root-meansquare deviation, thus, in certain areas, abrupt deviations in the speed and direction of movement of the working medium begin to occur, which can disrupt mixing or eliminate it.

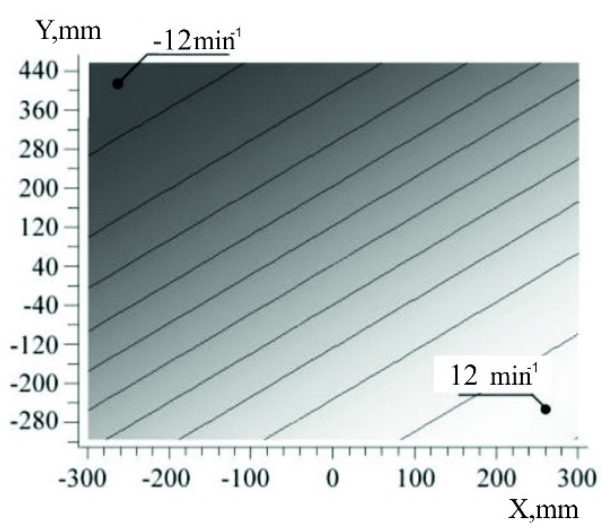

(a)

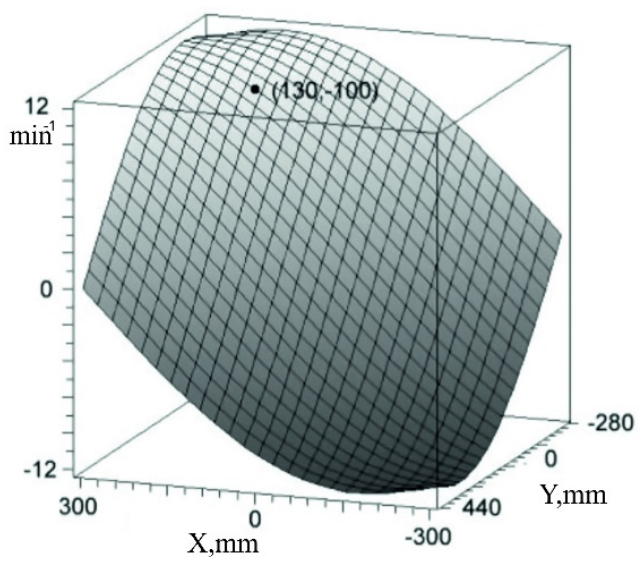

(b)

Fig. 2. Calculated values of the circulation velocity depending on the location of the vibration generator (a, b image in two and three-dimensional coordinate system, respectively)

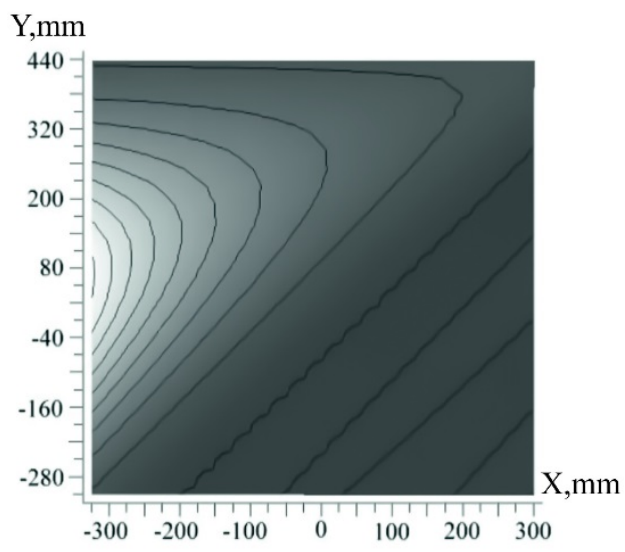

(a)

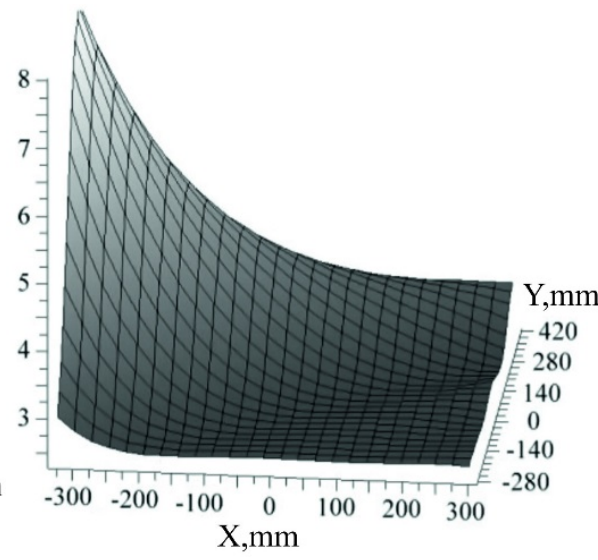

(b)

Fig. 3. Results of calculating the stability of the circulation motion, depending on the position of the vibration generator ( $\mathrm{a}, \mathrm{b}$ image in two and three-dimensional coordinate system, respectively) 
The fact is that in working medium with fine granulation, the different speed of parts displacement at different walls leads to a disruption of the circular motion and the separation of the flow of the working medium into several circulating or chaotically moving flows. This should be avoided, since in this case the treatment may not occur so evenly, the part can be tightened into the center of the container where the processing is less effective.

\section{Conclusion}

Taking into account the results of the studies conducted, as well as the influence of the location of the vibration generator on the useful power, the location of the vibration generator in the lower right corner, directly below the container wall at an angle of $45^{\circ}$ to the ordinate axis, perpendicular to the longitudinal axis, passing through the center of mass of the container is the most rational. This increase in productivity is provided both by increasing the useful power, and creating the conditions for a stable circulation of the mass of the load.

\section{References}

1. Perez J.P., Mécanique points matériels - solides - fluides (Masson, 1995)

2. R. Bridson, R. Fedkiw and J. Anderson, Robust treatment of collisions, contact and friction for cloth animation, , ACM Transactions on Graphics, v. 21, pp. 594-603 (2002).

3. A.N. Reznikov, Thermal processes in technological systems (Mechanical Engineering, Moscow, 1990)

4. N.S. Penkin, A.N. Penkin, V.M. Serbin, Fundamentals of tribology and tribotechnology (Mashinostroenie, Moscow, 2008)

5. V.B. Strutinskiy, V.M, Drozdenko, Dynamic processes in machine tools (Osnova-Print, Kiev, 2010)

6. V.F. Terentyev, Cyclic strength metallic materials (Mashinostroenie, Moscow, 2001)

7. C. Duan, M. Wang, Some metallurgical aspect of chips formed in high speed machining of high strength low alloy steel, J. Sci. Scripta Materialia. 52, pp.1001-1004 (2005)

8. J. Kopac, P. Krajnik, High-performance grinding - a review, J. Sci. Materials Processing Technology. 175, pp. 278-284 (2006)

9. T. Kuriyagawa, K. Syoji, H. Ohshita, Grinding Temperature within contact arc between whell and workpiece in high-efficiency grinding of ultrahard cutting tool materials, J. Sci. Materials Processing Technology. 136, pp. 39-47. (2003)

10. G. Gusanelli, A. Hessler-Wyser, F. Bobar, Microstructure at submicron scale of the white layer produced by EDM technique, J. Sci. Materials Processing Technology. 149 pp. 289-295 (2004)

11. Y.C. Fu, H.J. Xu, J.H. Xu, Optimization design of grinding wheel topography for high efficiency grinding, J. Sci. Materials Processing Technology. 129, pp. 118-122 (2002) 